\title{
Recurrent cerebral haemorrhage in a 65 year old man: advanced clinical neurology course, Edinburgh, 1997
}

\author{
Nicolas U Weir, Jan van Gijn, G Alistair Lammie, Joanna M Wardlaw, Charles P Warlow
}

Department of Clinical Neurosciences $\mathrm{N}$ U Weir J M Wardlaw

C P Warlow

Neuropathology

Laboratory,

Department of Clinical

Neurosciences,

Western General

Hospital, Crewe Road,

Edinburgh, UK

G A Lammie

Department of Neurology, University of Utrecht, Box 85500 , 350GA Utrecht, The Netherlands

$\mathrm{J}$ van Gijn

Correspondence to: Dr Nicolas U Weir, Department of Clinical Neurosciences, Western General Hospital, Crewe Road, Edinburgh EH4 2XU, Scotland, UK. Telephone 0044131343 6630; fax 0044131332 5150; email nuw@skull.dcn.ed.ac.uk

\section{Case presentation}

A 65 year old, right handed, retired police inspector presented to hospital as an emergency. That afternoon, while decorating, he had complained of a headache, although it did not prevent him from continuing with his work. Three hours later, however, his wife found him unable to speak, with a drooped right face and shortly afterwards, complete right sided paralysis. He was known to have hypertension and, 3 months earlier, he had been investigated for weight loss and painless obstructive jaundice. Endoscopic retrograde cholangiopancreatography (ERCP) had shown an impacted gall stone and appearances consistent with a periampullary tumour, although no histology was obtained. Sphincterotomy had led to improvement and the diagnosis had not been pursued further. Routine screening had also disclosed a lymphocytosis of $8.25 \times 10^{9} / 1$, which was confirmed to be due to an asymptomatic, early chronic lymphocytic leukaemia. His medications consisted of $100 \mathrm{mg}$ atenolol daily, $25 \mathrm{mg}$ mefruside daily, and $400 \mathrm{mg}$ cimetidine twice daily. He smoked 15 cigarettes a day and drank alcohol rarely.

On examination, he was drowsy with conjugate deviation of gaze to the left, and had dysphasia, a right homonymous hemianopia, and total right sided paralysis. Fundoscopy was normal. Other than mild jaundice, cardiovascular and general examination was normal. Brain CT (fig 1) showed a large, left frontoparietal haematoma with moderate mass effect and he had a leucocytosis of $21.2 \times 10^{9}$ (lymphocytes $9.75 \times 10^{9}$ ) with a normal haemoglobin and platelet count, and a bilirubin of 28 $\mathrm{mmol} / \mathrm{l}$.

After admission, his wife mentioned that he had been having word finding difficulties for about a year, although it was not clear if this had been progressive. After 6 weeks in hospital, he had recovered somewhat, although he still had dysphasia and right arm weakness. Repeat brain CT showed resolution of the haematoma and no underlying mass lesion. About a month later he had a generalised epileptic seizure and was started on $300 \mathrm{mg}$ phenytoin daily.

He remained stable for the next 20 months. Suddenly, however, his wife then noted that he could no longer plug in his shaver, screw the

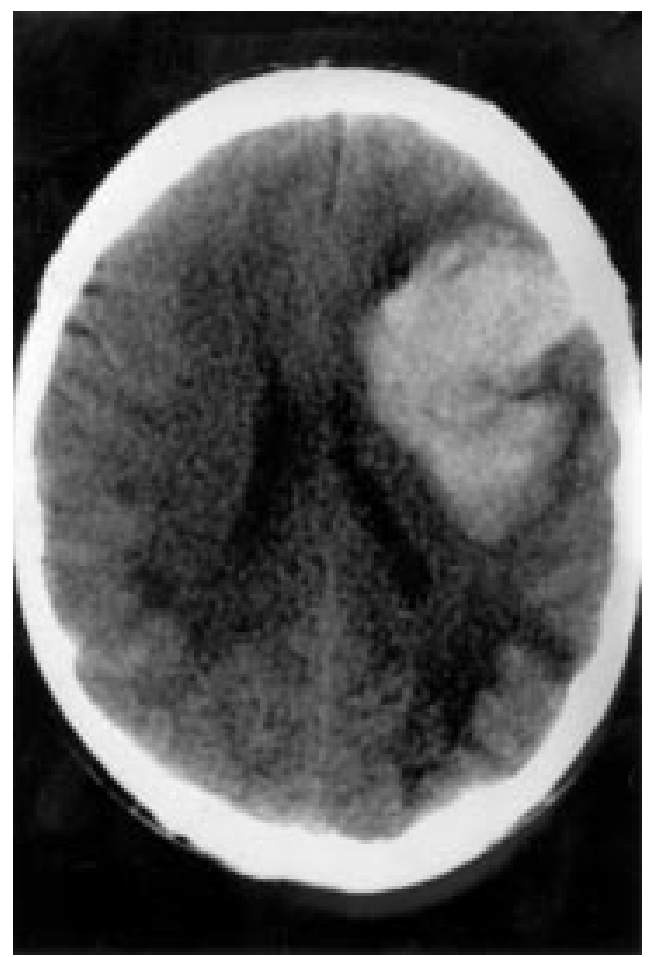

Figure 1 Brain CT (without contrast) on first presentation shows a large left frontoparietal haematoma (white) with some mass effect. Note that the haematoma extends out through the cortex to the surface of the brain.

toothpaste cap back on, or turn taps. Over the next 14 days his speech deteriorated and he had two generalised seizures. He then had a sudden episode of looking to the left with a vacant expression lasting some 2 to 3 minutes, after which he shuffled toward the door (he normally walked well) but could not locate the handle; he kept searching for it on the right side, seeming not to realise it was on the left. In his bedroom, he was unable to undress. The next morning, he was drowsy, incontinent, and occupying only the extreme right hand side of the bed.

On admission to hospital, he was pyrexial, confused, and variably cooperative, with a left homonymous hemianopia, dysphasia, and increased tone and hyperreflexia of the right side. General and cardiovascular examination, including the heart sounds, was normal. Brain 


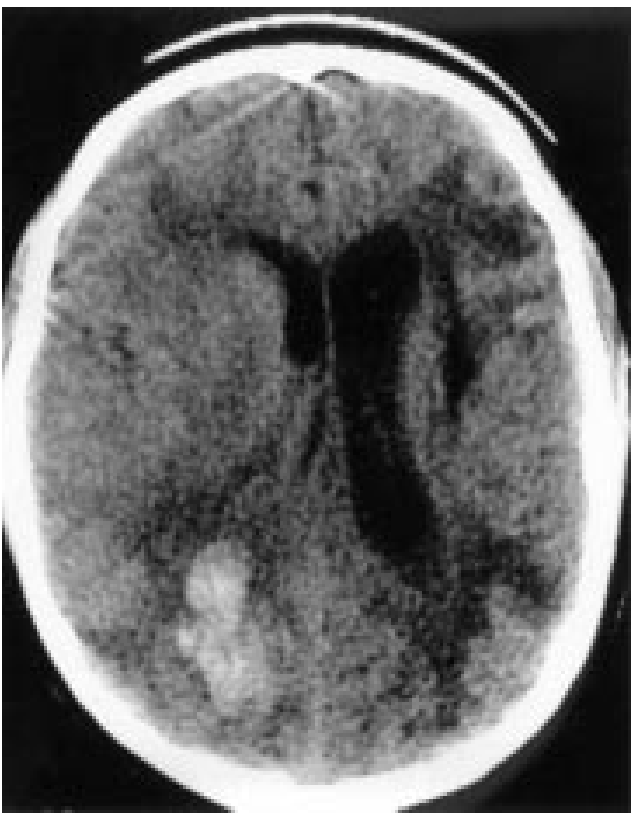

Figure 2 Brain CT (without contrast) 20 months after the first presentation. There is a new haematoma (white) in the right occipital region and a low density area in the left occipital region. The second suggests a further unrecognised stroke over the period after the first presentation. Note also the low density (dark) area in the left frontoparietal region with atrophy due to the original haematoma.

CT (fig 2) showed a right occipital haematoma with moderate mass effect, blood cultures grew coagulase negative staphylococci and $\alpha$ haemolytic streptococci, there was a leucocytosis of $31.9 \times 10^{9} / 1$ (lymphocytes $25.2 \times 10^{9} / 1$ ) with a normal haemoglobin and platelet count, normal clotting studies, and an erythrocyte sedimentation rate of $4 \mathrm{~mm} /$ hour. He became apyrexial and was stable for a few days, but then deteriorated, assuming a constant vacant expression, perseveration of speech, a shuffling gait, and constant turning to the right while walking. Repeat blood cultures grew coagulase negative staphylococci and gram negative bacilli. His leucocytosis had fallen to $23.7 \times 10^{9} / 1$ and $\mathrm{C}$ reactive protein was not raised. Despite his neurological dysfunction, he remained systemically well and his heart sounds were unaltered. His condition improved again over the next 10 days without specific intervention.

One evening, about 2 weeks later, he was found pale, clammy, and poorly responsive with a bitten lip. His right sided weakness was significantly worse although it was almost fully back to normal by the next day. Later that same day, however, he developed a pyrexia of $38.5^{\circ} \mathrm{C}$, a serum sodium of $125 \mathrm{mmol} / \mathrm{l}$, and a neutropenia of $1.7 \times 10^{9} / 1$ with neutrophil toxic granulation, haemoglobin of $10.2 \times 10^{12} / 1$ and a thrombocytopenia of $91 \times 10^{9} / 1$. Clotting studies disclosed an INR of 1.2 , activated partial thromboplastin time of 1.0 , and reduced fibrinogen concentration of $1.1 \mathrm{~g} / 1$. Despite intravenous co-amoxiclav he failed to improve and blood cultures were negative. Three days later he developed sudden onset of a dense right sided hemiparesis and tachypnoea, and brain CT showed two areas of haemorrhage in the left fronto-occipital and right parietooccipital areas (fig 3). By now, the fibrinogen

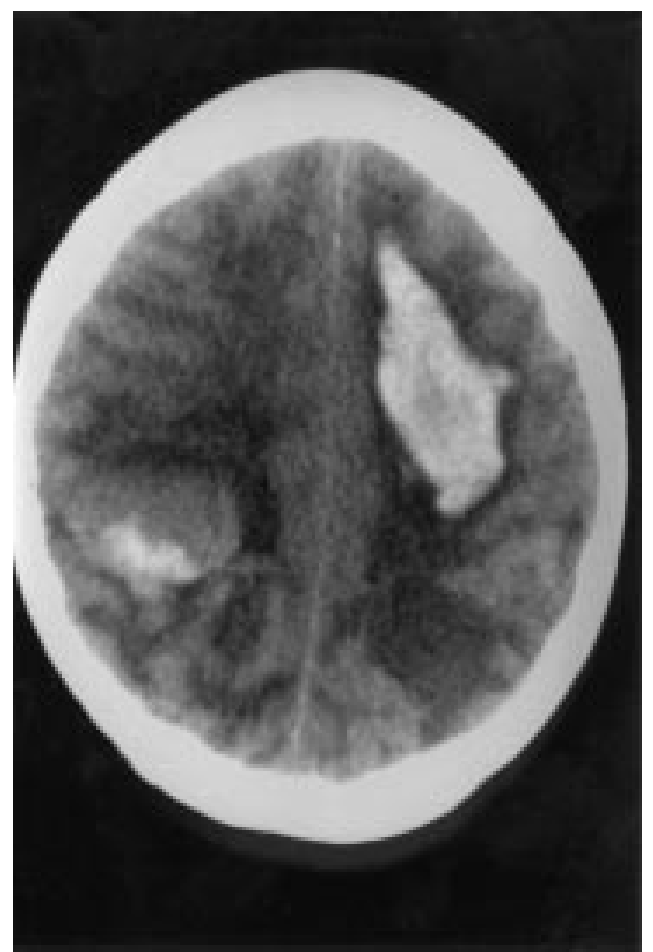

Figure 3 Brain CT 6 weeks after that in fig 2. There is a fresh haemorrhage in the left frontoparietal region and a subacute haemorrhage (mixed white and grey) in the right parietal region. In addition, there are older, resolving haemorrhages (dark grey) in the right and left occipital regions which must also have occurred after the brain CT in fig 2 .

concentration was further reduced to $0.7 \mathrm{~g} / 1$ and D-dimers were raised to 1.0 (range $0-0.5$ ). Two days later, splenomegaly was noted which, on ultrasound examination, measured $15 \mathrm{~cm}$ and was associated with extensive para-aortic lymphadenopathy, with a particularly large node by the pancreatic head.

His pyrexia eventually settled, and there was an unexpected improvement over the next 3 weeks. However, his general condition then declined and he died 2 weeks later. A postmortem was performed.

\section{Discussion}

Professor fan van Gijn

Firstly, I would like to separate this man's condition into episode 1 , the events of the presenting illness, and episode 2 , the events of the second and terminal illness whose separate parts I will call $2 \mathrm{a}, 2 \mathrm{~b}$, and $2 \mathrm{c}$. I will begin by reviewing the radiological findings and highlighting certain points. In episode 1 , the patient presented with features of a left hemispheric total anterior circulation stroke. The brain CT (fig 1) disclosed a rather dense, rather lateral, and sizeable haematoma extending up to the level of the horizontal part of the lateral ventricle with surrounding hypodensity and midline shift. The erythrocyte sedimentation rate was not provided and cerebral angiography was apparently not performed. His hypertension, chronic lymphocytic leukaemia, normal heart sounds, and uncertain periampullary pathology are noted. He made a moderate recovery, and, but for poststroke epilepsy, he was not 
troubled until episode 2, about 2 years later. It is this important interval that we must take account of when interpreting the underlying disease. At re-presentation (part 2a), there were clinical features of a lesion in the right parieto-occipital region and the brain CT (fig 2) shows an irregular haemorrhage in the right occipital lobe surrounded by a rim of hypodensity. Clinically, he was pyrexial on admission and blood cultures grew staphylococci, streptococci, and gram negative bacilli. $\mathrm{He}$ then entered part $2 \mathrm{~b}$ when he improved after a few days, then had a transient right hemiparesis, and then systemic illness with pyrexia, hyponatraemia, neutrophil toxic granulation and, with low fibrinogen and raised D-dimers, I presume disseminated intravascular coagulation. No CT was performed during this period. Episode 2c began 3 days later with sudden onset of a dense right hemiplegia after which he entered a terminal course. The final brain CT (fig 3) showed two new and very extensive areas of haemorrhage, firstly on the left, extending from the area of scarring related to episode 1 to the vertex, and, on the right, a further haematoma near that from episode $2 \mathrm{a}$. In fact, by this stage, the scan disclosed haemorrhages all over the brain.

Thus we must take account of a multiplicity of intracerebral haemorrhages, in two phases that are separated by a 2 year gap. We must also take account of the periampullary tumour, the chronic lymphocytic leukaemia, the pyrexia and positive blood cultures, and the disseminated intravascular coagulation. Lastly, the normality of the heart sounds has been emphasised repeatedly and this makes me suspicious. Indeed, this case greatly puzzles me, reminding me of Plato's cave, where people see only shadows and not the real world, and I have the impression that more things have been left out than given to me in the description of the case.

Firstly, the periampullary lesion; I will assume that because there was no surgical treatment, other investigations (that I have not been told about) showed local metastasis or infiltration of adjoining organs. The following 2 year survival virtually excludes pancreatic adenocarcinoma, in which median survival is only 5 months, and therefore a more benign process must be invoked. My tentative suggestion is an adenocarcinoma of the bile duct rather than the pancreatic duct, and given the prolonged survival, I suggest it was near the papilla.

Turning to the haematological problem, chronic lymphocytic leukaemia is a neoplasm of activated B lymphocytes and is often associated with hypogammaglobulinaemia and hence decreased resistance to encapsulated bacteria. In addition, the leukaemia is consistent with the splenomegaly and lymphadenopathy in the terminal phase of the illness. However, I suspect that the abdominal nodes were more likely to be related to the periampullary carcinoma as I would expect leukaemic infiltration to affect extra-abdominal nodes, and we are not told that this was the case. Leukaemia may also infiltrate the bone marrow and cause a pancytopenia, and indeed there was neutro- penia and thrombocytopenia at the time of the disseminated intravascular coagulation. However, there was no anaemia and the haematological abnormalities were related to the disseminated intravascular coagulation. Therefore, although the leukaemia may have been present in the bone marrow, I do not think that any infiltration was extensive and my impression is that the role of the leukaemia in the case was to predispose the patient to infection.

Turning now to the problem of multiple intracerebral haemorrhages, several possibilities come to mind. Of course, cerebral amyloid angiopathy is the commonest cause of multiple haemorrhages and certainly could be the underlying cause of this patient's condition. I am, however, put off this diagnosis principally because it seems far too simple for a clinicopathological case conference, and secondly because of the spatial distribution of the haemorrhages coupled with certain other features of the case (see below). Putting cerebral amyloid angiopathy on one side, we must then consider other causes of multiple intracerebral haemorrhages. Leukaemia certainly causes them, commonly due to blockage of the lumen of small vessels by leukaemic cells which invade, weaken, and ultimately rupture the vessel walls. However, this complication really only occurs in acute myeloid leukaemia and would be quite exceptional in chronic lymphocytic leukaemia. I therefore do not think we can implicate the leukaemia as the cause of the multiple intracerebral haemorrhages. The remaining causes to consider, then, are intracranial venous thrombosis, bacterial endocarditis (by means of arteritis secondary to septic emboli), thrombolytic treatment, disseminated intravascular coagulation, cerebral metastases (particularly melanoma), and necrotising systemic vasculitis.

That list covers the causes of multiple intracerebral haemorrhages in general. Let us now reconsider the scans. In episode 1 the site of the haematoma is in an arterial territory but is too lateral to be an ordinary, so called hypertensive intracerebral haemorrhage and it is too rostral to be related to a ruptured middle cerebral artery aneurysm. It would do for secondary haemorrhagic transformation of what could have been an infarct in the middle cerebral artery territory, except that the haematoma is too dense and, more importantly, the initial presentation was with a headache alone, which would be more in keeping with a primary haemorrhage. The next haemorrhage (episode 2a) is in the right occipital lobe and is also in an arterial territory. The two haemorrhages in the final phase of the illness, however, do not appear arterial. They appeared close together in time, perhaps even simultaneously (we cannot be sure because episode $2 \mathrm{~b}$ with worsening of the right hemiparesis was not followed up by a scan) and they were bilateral and parasagittal. These bleeds are different from the first haemorrhages, and for these reasons I suspect they may have been of venous origin.

With this in mind, let us again explore the remaining causes of multiple intracerebral haemorrhages. We have discounted cerebral amyloid angiopathy and leukaemia, and there 


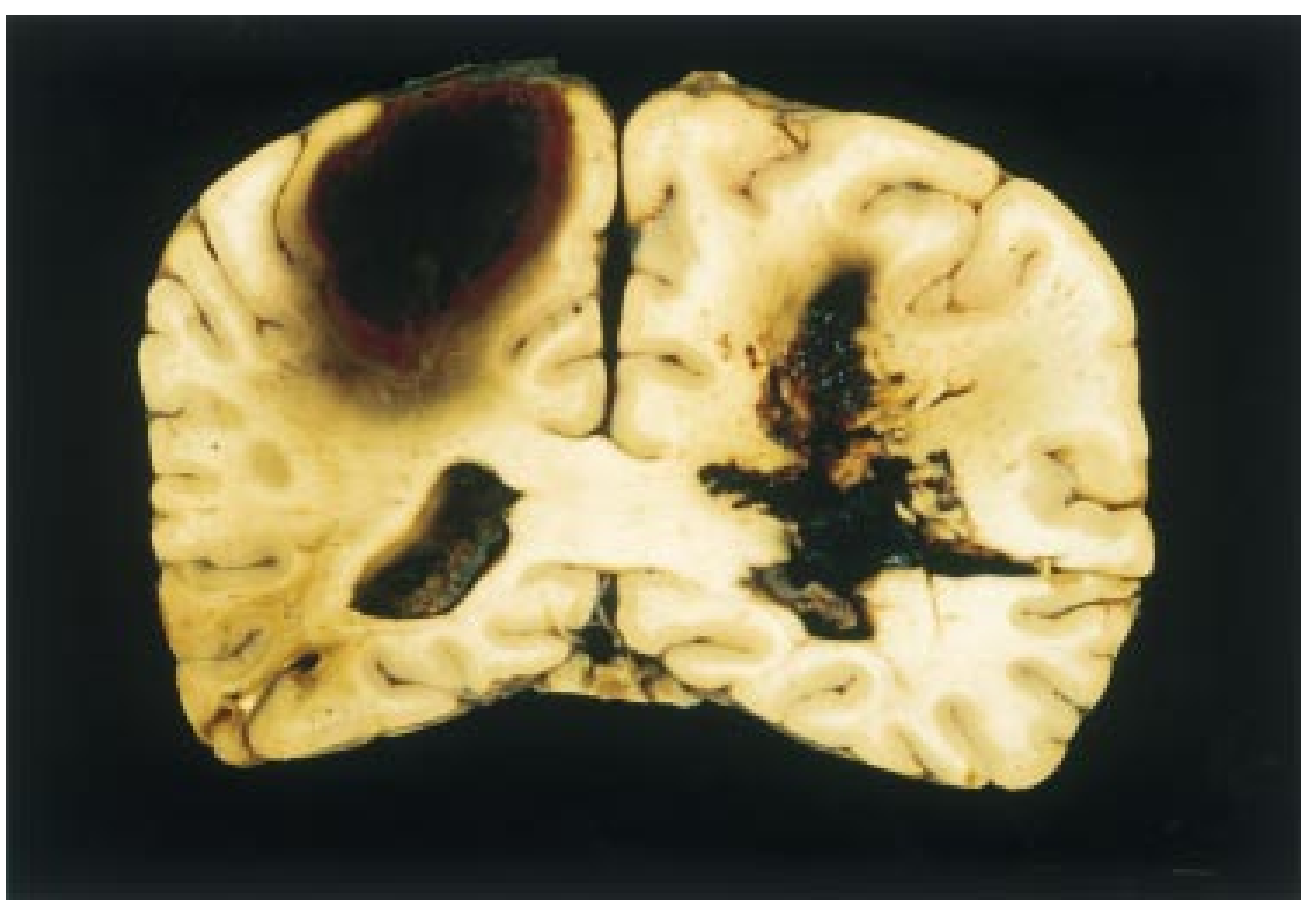

Figure 4 Coronal whole brain section through the splenium of the corpus callosum. The large lobar haematoma in the left hemisphere shows subarachnoid extension on a broad front, and the irregular right sided subcortical bleed has ruptured into the lateral ventricle. There are several small cortical and subcortical infarcts at the inferolateral angle of the left hemisphere.

is no evidence to support disseminated intravascular coagulation, metastases, or necrotising systemic vasculitis as the cause of the haemorrhages in the first phase of the disorder. What about the possibility of endocarditis with rupture of vessels secondary to septic emboli? The ERCP investigation 3 months before episode 1 is a recognised cause of systemic sepsis, often with enterococci. The chronic lymphocytic leukaemia might have predisposed to infection with just such bacteria because of the associated hypogammaglobulinaemia. Thus there are reasonable grounds to consider the possibility of endocarditis. Against this, however, are the normal heart sounds (though in $20 \%-30 \%$ of cases the heart valves are normal, at least to start with) and the absence of evidence for pyrexia, raised erythrocyte sedimentation rate or positive blood cultures during episode 1 . Also, to explain the 2 year interval, I have to assume either that he was given some treatment I was not told about or there was some spontaneous healing precisely because the heart valves were normal. Clearly, we are on thin ice here as Osler's triad for the diagnosis of endocarditis requires hemiplegia, fever, and a murmur and we have only the hemiplegia and no fever or murmur.

What about the occipital haemorrhage in phase 2a? Could that have been a mycotic aneurysm which had been left over from the first phase? Certainly they have been recorded as a cause of stroke up to 6 months after adequate treatment for endocarditis and noted to persist on cerebral angiograms for up to 2 years. Alternatively, could there have been a second episode of endocarditis with arteritis which would be consistent with the positive blood cultures and fever in the second episode? Again, we are left with the worryingly normal heart sounds and erythrocyte sedimentation rate, but the second might have related to the low plasma fibrinogen concentration.

Now we need to account for what I have interpreted as a couple of venous haemorrhages in phase $2 \mathrm{c}$. I think that there are two likely causes in this patient. Firstly, there was the disseminated intravascular coagulation and secondly, they might have been secondary to an intracranial venous thrombosis. Indeed, it is conceivable that both conditions may have coexisted. Certainly they are both associated with sepsis and with widespread malignancy. A more remote possibility is non-bacterial thrombotic endocarditis. This condition is often the cause of stroke in patients with terminal disorders and is particularly associated with disseminated intravascular coagulation and carcinoma. However, I do not think that we can implicate it here as I would expect it to produce infarcts (which may then become haemorrhagic) only in arterial territories.

Therefore, my diagnosis of the cause of the first two intracerebral haemorrhages is infective endocarditis with cerebral arteritis, on a background of normal or nearly normal heart valves. In the terminal phase of the illness there was disseminated intravascular coagulation and probably intracranial venous thrombosis which conspired to cause the venous haemorrhages in phase 2 c. In addition, there was an adenocarcinaoma of the bile duct with nodal metastases and chronic lymphocytic leukaemia without marked infiltration of the bone marrow.

\section{PATHOLOGY}

Dr Alistair Lammie

Postmortem was performed one day after death. The heart weighed $400 \mathrm{~g}$ and showed moderate left ventricular hypertrophy, consist- 


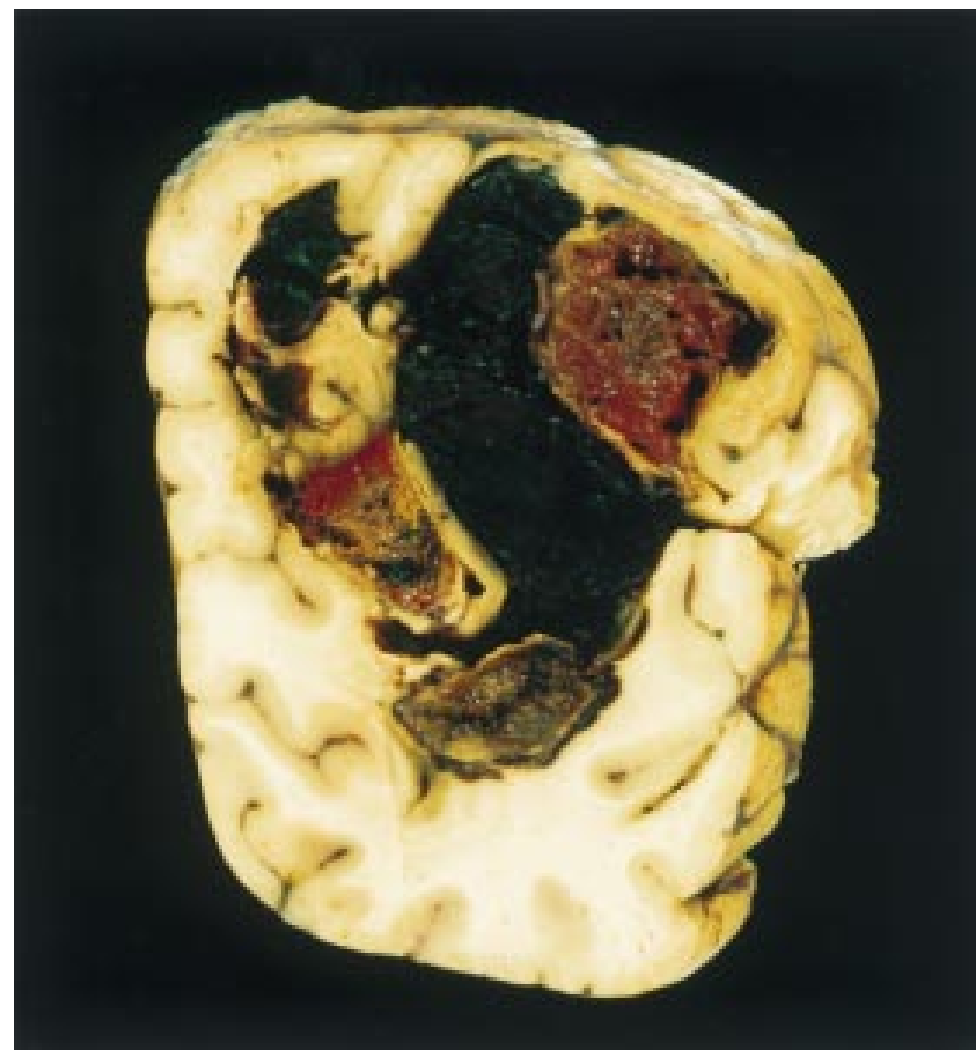

Figure 5 Right occipital lobe: a dark central, predominantly subcortical haematoma of recent onset, surrounded by smaller bleeds showing the variegated hues of blood breakdown products, suggestive of recurrent episodes of rebleeding.

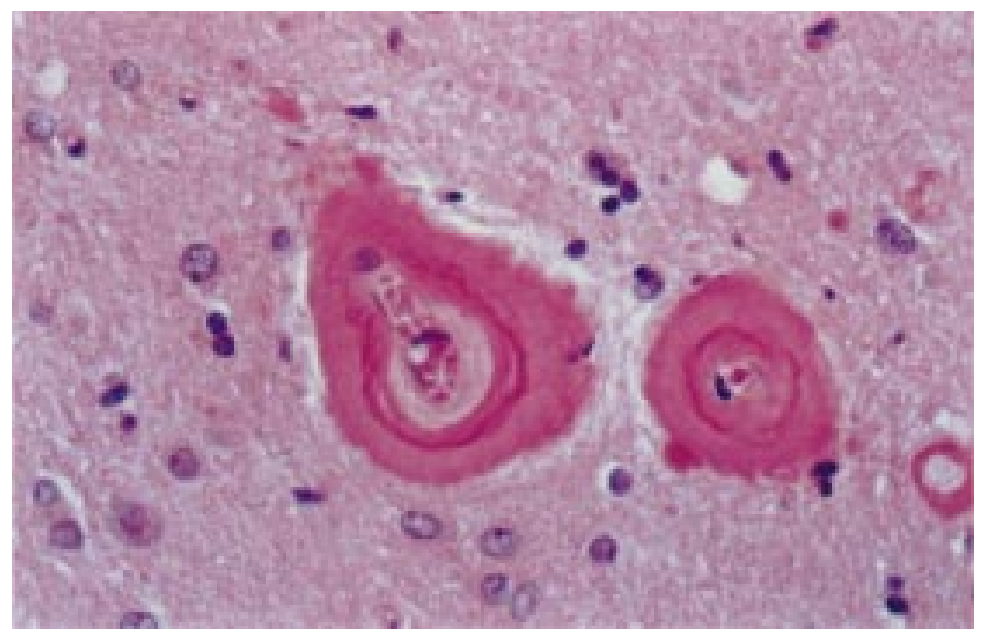

Figure 6 Concentric acellular, eosinophilic thickening of penetrating cortical vessels with severe luminal narrowing, typical of advanced amyloid angiopathy (haematoxylin and eosin, originally $\times 450$ ). chronic lymphocytic leukaemia, with evidence of leukaemic infiltration in the bone marrow, thyroid, kidney, and liver. There was no intrinsic ampullary tumour and the pancreas was normal. The cause of the painless jaundice was a hard, $5 \mathrm{~cm}$ diameter lymph node compressing the distal end of the common bile duct.

The brain was globally swollen, weighed $1598 \mathrm{~g}$, and externally bore multiple small areas of subarachnoid blood, both old and recent. There was an incidental unruptured aneurysm on the bifurcation of the left middle cerebral artery. Coronal brain slices confirmed multiple intracerebral haemorrhages of varying age. Some communicated with the ventricles but many were superficial lobar bleeds with varying degrees of cortical, subarachnoid, and subdural extension. In some there was evidence of rebleeding (figs 4 and 5). The number of bleeds was greatest in the parieto-occipital lobes, less in the frontotemporal area.

Histology disclosed a widespread, "smudgy", hyaline, eosinophilic, and diffuse thickening of vessel walls, affecting predominantly the medium and small sized arteries in the cortex and leptomeninges (fig 6), but sparing the white matter. Some double barrelled walls were seen. These appearances are characteristic of cerebral amyloid angiopathy, presumably sporadic, and were confirmed by Sirius red histochemical and $\beta \mathrm{A} 4$ amyloid antibody immunohistochemical staining (fig 7). The second also disclosed scattered neocortical amyloid plaques.

Although amyloid deposition was also seen in some leptomeningeal veins, there was no obvious dural venous sinus or leptomeningeal vein thrombosis and no histological features to suggest haemorrhagic venous infarction. A few amyloid laden vessels were associated with a perivascular, chronic inflammatory infiltrate, but there was no evidence of vasculitis. The cortex harboured scattered microinfarcts, but there was no evidence of large vessel regional infarction. Characteristically, there was also a significant non-amyloid vasculopathy in the deep white matter, typical of age and hypertension related arteriolosclerosis ("mixed microangiopathy"). The associated white matter was considerably rarefied, gliotic, and focally cavitated.

It is perhaps useful to ask why this patient had multiple spontaneous cerebral bleeds, for up to one half of asymptomatic, elderly people without ischaemic or haemorrhagic lesions show evidence of cerebral amyloid angiopathy at postmortem. ${ }^{1-4}$ It is suggested that amyloid laden vessels are brittle and do not withstand changes in pressure as well as non-amyloid laden vessels. ${ }^{5}$ Certainly, the number and location of bleeds (and infarcts) correlates with the distribution of cerebral amyloid angiopathy and with the density of the affected vessels. ${ }^{35}$ However, whereas amyloid laden vessels are restricted to the cortex and leptomeninges, several bleeds seemed, in this brain, to reside solely within white matter. It is therefore likely that the non-amyloid, deep small vessel disease, which in my experience is an invariant feature of advanced cerebral amyloid angiopa- 


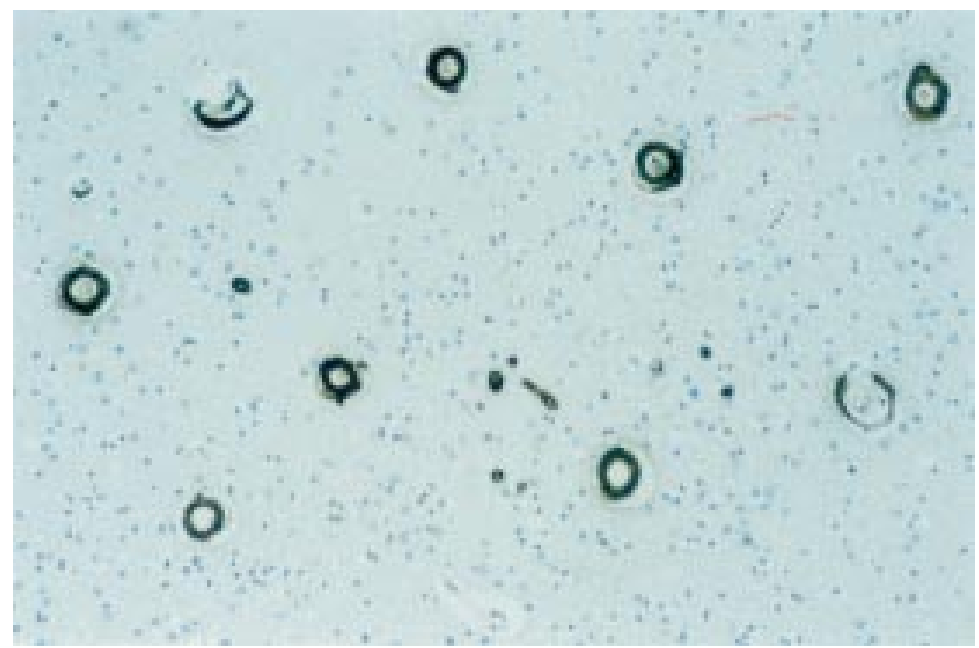

Figure 7 Immunohistochemical demonstration of cortical $\beta$ A4-amyloid angiopathy ( $\beta A 4$ antibody; originally $\times 120$ ). thy, may be the source of a proportion of haemorrhages in this condition.

It has been said that hypertension is "neither necessary nor sufficient for subsequent primary intracerebral haemorrhage". ${ }^{6}$ Indeed, most people with hypertension never develop a cerebral haemorrhage and presumably there must be additional factors at work in those that do. The same is likely to be true of cerebral amyloid angiopathy. Did other factors contribute to the intracranial haemorrhages in this man? Firstly, he had hypertension, a well established risk factor in its own right. Secondly, he had chronic lymphocytic leukaemia with evidence of bone marrow infiltration, although the contribution of this to the bleeding is uncertain. Classically, acute lymphoblastic ${ }^{7}$ and acute promyelocytic leukaemias ${ }^{8}$ are associated with multiple lobar intracerebral haemorrhages, but then normally with a very low platelet count or blast crisis, neither of which were present in this patient. Lastly, there was sepsis and disseminated intravascular coagulation in the terminal phase of the illness. Clearly then, there were other factors contributing to the repeated cerebral bleeds.

Although this patient did not present with cognitive decline, many patients with cerebral amyloid angiopathy present with dementia of either acute or progressive onset. ${ }^{1259}$ This dementia is probably multifactorial, with Alzheimer-like pathology, the subcortical leukoencephalopathy, multiple haemorrhages, and cortical infarcts all contributing. This patient had a typical pattern of Alzheimer-like pathology with numerous amyloid plaques but only modest numbers of neurofibrillary tangles, all of which were restricted to the hippocampus. ${ }^{510} 11$ Therefore, on purely pathological grounds, these features are insufficient to support a neuropathological diagnosis of Alzheimer's disease, but may represent an early stage in its neuropathological evolution. Perhaps patients with severe cerebral amyloid angiopathy die prematurely, whereas patients with less severe vascular involvement live longer, develop more Alzheimer pathology, and become demented. ${ }^{12}$

\section{Conclusion}

Professor $\mathcal{F}$ van Gijn's diagnosis

Bacterial endocarditis with cerebral arteritis Venous haemorrhage secondary to disseminated intravascular coagulation and intracranial venous thrombosis

Chronic lymphocytic leukaemia

Adenocarcinoma of the bile duct

Pathological diagnosis

Sporadic cerebral amyloid angiopathy

Chronic lymphocytic leukaemia

Bronchopneumonia and pulmonary emboli

\section{Comment}

It has been estimated that sporadic cerebral amyloid angiopathy accounts for about $10 \%$ of all cases of primary intracerebral haemorrhage. ${ }^{13}$ Although a definitive diagnosis of cerebral amyloid angiopathy can only be made with confidence at postmortem or by brain biopsy, the clinical and radiological features may strongly suggest the diagnosis during $\operatorname{life}^{13}$ : Radiologically, cerebral amyloid angiopathy should always be considered when a patient presents with multiple, lobar intracerebral haemorrhages of simultaneous or sequential onset. The haemorrhage, whether single or multiple, is usually rather large and "dilute" in appearance, with surrounding low density (oedema) and often a fluid level, all at a stage in the evolution of the lesion where a focal, dense lesion would be expected, without oedema or a fluid level, were it a "hypertensive" bleed. Indeed, haemorrhages related to cerebral amyloid angiopathy often have a similar appearance to those seen in patients on anticoagulant drugs or with a coagulopathy. In addition, the haemorrhages are usually peripheral and may extend to the cortical or ventricular surface and thence into the subarachnoid space. There is often also evidence on the scan of a simultaneous or previous haemorrhagic lesion elsewhere in the brain (particularly with MRI, which is more specific for prior haemorrhage) and a careful search for this is advisable.

Two characteristic clinical features should also call the diagnosis of cerebral amyloid angiopathy to mind. ${ }^{9}$ Firstly, patients may experience recurrent brief focal motor, visual, or sensory neurological symptoms. The sensory symptoms occasionally have a spreading quality that suggests a focal seizure, although the pathophysiology of all the transient phenomena remains uncertain. Secondly, and as noted above, patients may present with cognitive decline. The combination of two or more features of the triad of lobar haemorrhage, transient neurological symptoms, and cognitive impairment should particularly raise suspicion of cerebral amyloid angiopathy. However, as this case illustrates, the condition continues to be underdiagnosed clinically. ${ }^{12}$

The importance of antemortem diagnosis is, in any case, somewhat limited at present. None the less, it does provide the opportunity to exclude other treatable causes of primary intracerebral haemorrhage, to exclude the rare familial forms of cerebral amyloid angiopathy and possibly to advise against brain surgery for fear of precipitating haemorrhage. ${ }^{14}$ More 
importantly, however, as more is learned of the pathophysiology and molecular pathogenesis of cerebral amyloid angiopathy, it may not be long before specific preventive and therapeutic intervention is possible.

The weight of in vivo and in vitro evidence presently favours the vascular smooth muscle cell as the source of vascular amyloid, ${ }^{15-18}$ and also as the target of subsequent amyloid mediated toxicity. ${ }^{1920}$ Whereas the amyloidogenic protein in the Icelandic variant of hereditary cerebral amyloid angiopathy is cystatin $\mathrm{C},{ }^{21}$ in all other forms of cerebral amyloid angiopathy, including sporadic and the Dutch familial variants, the protein is $\beta$-amyloid, which is itself derived from cleavage of the $\beta$-amyloid protein precursor ( $\beta$-APP), encoded on the long arm of chromosome $21 .^{22}$ Dutch-type hereditary cerebral amyloid angiopathy is associated with mutations of the $\beta$-APP gene, ${ }^{23}{ }^{24}$ but no such mutations have been so far described in sporadic cerebral amyloid angiopathy. ${ }^{25}$ Attention has instead focused on the possible role of the Apo-E genotype ${ }^{26-28}$ and of other amyloid binding proteins, such as amyloid $\mathrm{P}$ component, $\quad \alpha 1$-antichymotrypsin and proteoglycans. ${ }^{12}$ It seems likely that cerebral amyloid angiopathy represents a manifestation of the aging cerebral vascular tree in genetically predisposed people, and may be the consequence of a complex variety of gene expression and post-translational processing events that are at present incompletely understood.

Finally, and as Professor van Gijn suggested, it is worth recalling that common disorders do indeed occur commonly. This useful rule of thumb seems to apply not only in everyday practice, but sometimes even in a clinicopathological conference.

We are grateful to Dr MS Dennis for permission to report this case. NUW is supported by a Wellcome Research Training Fellowship and GAL and JMW by the MRC.

1 Esiri MM, Wilcock GK. Cerebral amyloid angiopathy in dementia and old age. $\mathcal{F}$ Neurol Neurosurg Psychiatry 1986;49:1221-6.

2 Masuda J, Kenzo T, Ueda K, et al. Autopsy study of incidence and distribution of cerebral amyloid angiopathy in Hisayama, Japan. Stroke 1988;19:205-10.

3 Vonsattel JPG, Myers RH, Hedley-White ET, et al. Cerebral amyloid angiopathy without and with cerebral haemorrhages: a comparative histological study. Ann Neurol 1991;30:637-49

4 Yamada M, Tsukagoshi H, Otomo E, et al. Cerebral amyloid angiopathy in the aged. 7 . Neurol 1987;234:371-6.

5 Vinters HV. Cerebral amyloid angiopathy. A critical view. Stroke 1987;18:311-24.
6 Warlow CP, Dennis MS, van Gijn J, et al. Stroke. A practical guide to management. Chapter 8. Oxford: Blackwell Science, 1996:287-8.

7 Groch SN, Sayre GP, Heck FJ. Cerebral haemorrhage in leukaemia. Arch Neurol 1960;2:439-51.

8 Graus F, Rogers LR, Posner JB. Cerebrovascular complications in patients with cancer. Medicine 1985;64:16-35.

9 Greenberg SM, Vonsattel JPG, Stakes JW, et al. The clinical spectrum of cerebral amyloid angiopathy: presentations without lobar haemorrhage. Neurology 1993;43:2073-9.

10 Gilbert JJ, Vinters HV. Cerebral amyloid angiopathy: incidence and complications in the ageing brain. I. Cerebral haemorrhage. Stroke 1983;14:915-23.

11 Vinters HV, Gilbert JJ. Cerebral amyloid angiopathy: incidence and complications in the ageing brain. II. The distribution of amyloid vascular changes. Stroke 1983;14: 924-8.

12 Coria F, Rubio I. Cerebral amyloid angiopathies. Neuropathol Appl Neurobiol 1996;22:216-27.

13 Warlow CP, Dennis MS, van Gijn J, et al. Stroke. A practical guide to management. Chapter 8. Oxford: Blackwell Science, 1996:294-5.

14 Torack RM. Congophilic angiopathy complicated by surgery and massive haemorrhage. Am f Pathol 1975;81: 349-66.

15 Coria F, Moreno A, Torres A, et al. Distribution of Alzheimer's disease and amyloid protein precursor in normal human and rat nervous system. Neuropathol Appl Neurobiol 1992;18:27-35.

$16 \mathrm{Ko} \mathrm{L}$, Sheu KR, Blas JP. Immunohistochemical colocalisation of amyloid precursor protein with cerebrovascular amyloid in Alzheimer's disease. Am $\mathcal{f}$ Pathol 1991;139:523-33.

17 Shoji M, Hirai S, Harigaya Y, et al. The amyloid $\beta$-protein precursor is localised in smooth muscle cells of leptomeningeal vessels. Brain Res 1990;530:113-6.

18 Wisniewski HM, Wegiel J, Wang KC, et al. Ultrastructural studies of the cells forming amyloid in the cortical vessel wall in Alzheimer's disease. Acta Neuropathol 1992;84:11727

19 Kawai M, Kalaria R, Cras P, et al. Degeneration of vascular muscle cells in cerebral amyloid angiopathy of Alzheimer's disease. Brain Res 1993;623:142-6.

20 Thomas T, Thomas G, McLendon C, et al. $\beta$-amyloidmediated vasoactivity and vascular endothelial damage. Nature 1996;380:168-71.

21 Abrahamson M, Jonsdottir S, Olafsson I, et al. Hereditary cystatin C amyloid angiopathy: identification of the disease-causing mutation and specific diagnosis by polymerase chain reaction based analysis. Hum Genet 1992;89:377-80.

22 Selkoe DJ. Cell biology of the amyloid $\beta$-protein precursor and the mechanism of Alzheimer's disease. Anпи Rev Cell Biol 1994;10:373-403.

23 Hendriks L, van Duijn CM, Cras P, et al. Presenile dementia and cerebral haemorrhage linked to a mutation at codon 692 of the $\beta$-amyloid precursor protein gene. Nat Genet 1992;1:218-21.

24 Levy E, Carman MD, Fernandez-Madrid EJ, et al. Mutation of Alzheimer's disease amyloid gene in hereditary cerebral haemorrhage Dutch type. Science 1990;248:1124-6.

25 Graffagnino C, Herbstreith MH, Roses AD, et al. A molecular genetic study of intracerebral haemorrhage. Arch Neurol 1994;51:981-4.

26 Strittmatter WJ, Weisgraber KH, Huang DY, et al. Binding of human apolipoprotein $\mathrm{E}$ to synthetic amyloid $\beta$ peptide: soform specific effects and implications for late-onset Alzheimer's disease. Proc Natl Acad Sci 1993;90:8098-102.

27 Greenberg SG, Rebeck GW, Vonsattel JPG, et al. Apolipoprotein E $\& 4$ and cerebral haemorrhage associated with amyloid angiopathy. Ann Neurol 1995;38:254-9.

28 Nicoll JAR, Burnett C, Love S, et al. High frequency of apolipoprotein E $\varepsilon 2$ in patients with cerebral haemorrhage due to cerebral amyloid angiopathy. Ann Neurol 1996;39: $682-3$. 